\title{
A Badging System for Reproducibility and Replicability in Remote Sensing Research
}

\author{
Alejandro C. Frery ${ }^{\circledR}$, Senior Member, IEEE, Luis Gomez ${ }^{\circledR}$, Senior Member, IEEE, and Antonio C. Medeiros ${ }^{\circledR}$
}

\begin{abstract}
Remote Sensing is both an active research area and the source of valuable information for decision-making. Many actors play a fundamental role in Remote Sensing, from industry (public or private) to large or small research groups. From that intensive activity, methods, algorithms, and techniques are continuously published or broadcasted through papers, conference presentations, repositories, patents, standards, and other means. The consumers of that information need it to be readily available and dependable. Reproducible research can handle those needs. In this article, we discuss two concepts: reproducibility and replicability in the context of Remote Sensing research. We propose a badging system suited to the specifics of the Remote Sensing community. Such a system aims at both recognizing the level of the reproducibility of the research, and to help increase its visibility. We show examples of reproducible research and provide clues to make easier the transition to the inevitable new times that embrace contemporary science and technology.
\end{abstract}

Index Terms-Remote sensing, replicability, reproducibility.

\section{RATIONALE}

$\mathbf{R}$ EPRODUCIBILITY is at the core of experimental sciences. It is also a basilar element of scientific integrity. The advent of data science is leading to new requirements and practices able to cope with the challenges posed by vast volumes of data, often of dynamic nature.

New grounds for Reproducible Research were set before the widespread use of deep learning and other massively data-based techniques.

Reproducible research has many benefits but also many challenges, and the ones related to software are of utmost importance.

Most Remote Sensing papers support the evidence of the proposed research by computational codes of medium or high complexity. More often than not, while not bearing in mind the reproducibility of the research done, codes are developed under

Manuscript received April 30, 2020; revised August 14, 2020; accepted August 22, 2020. Date of publication August 25, 2020; date of current version September 7, 2020. This work was supported in part by the Conselho Nacional de Desenvolvimento Científico e Tecnológico (Brazil) and in part by Fundação de Amparo Pesquisa do Estado de Alagoas (Brazil). (Corresponding author: Alejandro C. Frery.)

Alejandro C. Frery is with the School of Mathematics and Statistics, Victoria University of Wellington, Wellington 6140, New Zealand, and also with the Key Laboratory of Intelligent Perception and Image Understanding of the Ministry of Education, Xidian University, Xi' an 710126, China (e-mail: acfrery@gmail.com).

Luis Gomez is with the Universidad de Las Palmas de Gran Canaria, 35017 Las Palmas de Gran Canaria, Spain (e-mail: luis.gomez@ulpgc.es).

Antonio C. Medeiros is with the Laboratório de Computação Científica e Análise Numérica, Universidade Federal de Alagoas, Maceió 57072-900, Brazil (e-mail: antoniomedeiros@laccan.ufal.br).

Digital Object Identifier 10.1109/JSTARS.2020.3019418 the philosophy of "getting just results for the paper," and then, they will rest in hard drives. It is frequent that even the ones that implemented the codes cannot execute them after some time has passed.

Among the efforts made toward building Remote Sensing Reproducible Research (RSRR) environments, one may mention Code Ocean and GRSS Remote Sensing Code Library. These two initiatives belong to the IEEE scientific ecosystem.

However, is this enough for sound Science in Remote Sensing? We do not think so. Balz and Rocca [1] conducted an online survey focused on experienced scientists, including editors and associate editors, that are active in the scientific publication process in the field of Synthetic Aperture Radar Remote Sensing. With 250 responses, of which only one did not complete the survey, they concluded that $75 \%$ of the respondents encountered problems when trying to reproduce or replicate results from works published in some of the major journals of the area.

Many authors continuously strive to disseminate their research findings in such a way that any user will be able to validate them at a later stage. Those ways include the proposal of software architectures, the use of open data, Free/Libre Open Source Software (FLOSS), and other initiatives.

Barba [2] identifies several usages of the terms "reproducibility" and "replicability" and also the emergence of the term "repeatability," among others. The work by Fidler and Wilcox [3] discusses the use of those terms, surveys meta-analyses that characterize the reproducibility crisis, addresses epistemological aspects of reproducibility and its value in science, and, finally, comments upon initiatives to alleviate such crisis.

We will discuss two core concepts: reproducibility and replicability in the context of Remote Sensing research. Such simplification aims at arriving at the suggestion of good scientific practices and a badging system for the Remote Sensing publication ecosystem.

We will use the metaphor of dwarfs standing on the shoulders of giants, which, in the words of Isaac Newton [4], is:

If I have seen further, it is by standing on the shoulders of Giants.

Reproducibility consists of allowing the whole community to reach the researcher's shoulders. Replicability grants the community to stand on the researcher's shoulders.

Scientific work is reproducible if other researchers can obtain the data and the code and, effortlessly, obtain the same products (analyses and reports). Scientific work is replicable if it reported in such a way that other researchers can perform similar studies and arrive at compatible conclusions. 


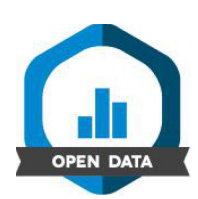

(a)

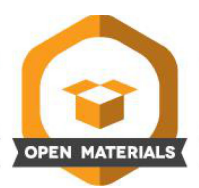

(b)

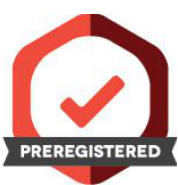

(c)

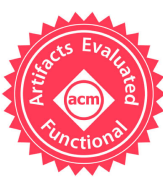

(a)

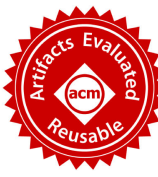

(b)

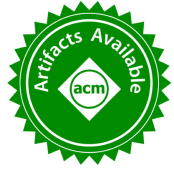

(c)

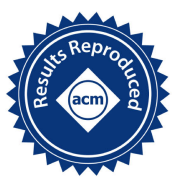

(d)

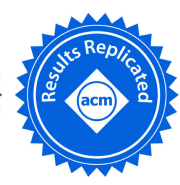

(e)

Fig. 1. Open science three-level badging system. (a) Open Data. (b) Open Materials. (c) Preregistered.

\title{
Quoting Ref. [5]:
}

a promise in a published paper to make code and/or data "available upon request" is not a reproducible practice: digital artifacts should already be in a suitable repository.

We, thus, believe that the Remote Sensing community needs to adhere to good practices of reproducibility and replicability. In this line, we propose a badging system that recognizes works that, fully or partially, adhere to such practices.

The remainder of this article analyzes a journal, a scientific society, and a platform that, implicitly or explicitly, use badging systems (see Section II). Section III assesses three platforms available through IEEE that promote reproducibility, but not the point of leading to Remote Sensing fully reproducible research. Then, in Section IV, we propose a badging system that, although instantiated for the IEEE Geoscience and Remote Sensing journals, could be applied to other venues. Section V briefly describes tools that may support this initiative from the researchers' side. Section VI comments three of the major challenges this initiative may face.

\section{BADGING SYSTEMS}

The Image Processing On Line (IPOL) journal [6] is a noteworthy example of reproducibility and replicability. In its own words, IPOL

\begin{abstract}
is a research journal of image processing and image analysis which emphasizes the role of mathematics as a source for algorithm design and the reproducibility of the research. Each article contains a text on an algorithm and its source code, with an online demonstration facility and an archive of experiments. Text and source code are peer-reviewed, and the demonstration is controlled. IPOL is an Open Science and Reproducible Research journal.
\end{abstract}

We notice, though, that publishing in IPOL requires more work than publishing in other journals. The journal imposes strict conditions on the source code that, more often than not, take much time from the authors. Going down such a path does not seem adequate for the Remote Sensing community.

The scientific community is striving to recognize those articles that comply with reproducibility criteria by assigning badges. In the following, we mention two of those initiatives.

To date, 67 journals use the Open Science badging system. ${ }^{1}$ It consists of three levels: (a) Open Data, (b) Open Materials, and (c) Preregistered study (cf. Fig. 1). According to Munafò et al. [7], this practice increased by order of magnitude articles with open data in the Psychological Science journal.

\footnotetext{
${ }^{1}$ [Online]. Available: https://cos.io/our-services/open-science-badges/
}

Fig. 2. ACM five-level badging system. (a) Functional artifacts. (b) Reusable artifacts. (c) Available artifacts. (d) Reproducible results. (e) Replicable results.

The Association for Computing Machinery (ACM) has a fivelevel badging system, ${ }^{2}$ illustrated in Fig. 2.

It is worth noting that levels in Fig. 2(a) and (b) do not grant per se reproducibility. They just acknowledge that the authors made their code available to reviewers, and that it is functional and reusable. The Remote Sensing community should strive to attain, at least, level in Fig. 2(d).

We believe that a similar, even if more straightforward, badging system will have a great positive impact in the Remote Sensing community.

\section{PLATFORMS}

Konkol et al. [8] discuss ten infrastructures for reproducible practices. The authors perform analyses from the viewpoints of publishers, editors, authors, readers and reviewers, and librarians. The work is an authoritative reference for choosing among the currently available options.

In the following, we will comment on two different approaches, both available through IEEE.

\section{A. Code Ocean}

\section{IEEE has teamed with Code Ocean to ${ }^{3}$}

enable authors to further enhance the visibility and impact of their research by enabling them to share their code on Code Ocean so that readers can browse, view, run and experiment with the code.

Readers can discover, browse, run, modify code, and input data to experiment, reproduce, and build on your research, all in the cloud, without any other setup or software license.

Code Ocean currently supports $\mathrm{C} / \mathrm{C}++$, Fortran, Java, Julia, Lua, MATLAB, Octave, Perl, Python, R, and Stata in most available versions. It, thus, covers the major programming languages in which the Remote Sensing community develops its research.

The service is available to authors of already accepted papers, so it cannot be integrated into the review process.

\section{B. Data Port}

IEEE describes DataPort as ${ }^{4}$

a valuable and easily accessible data platform that enables users to store, search, access, and manage data. The data platform is designed to accept all formats and sizes of datasets (up to 2T), and it provides both downloading capabilities and access to datasets in the Cloud.

\footnotetext{
${ }^{2}$ [Online]. Available: https://www.acm.org/publications/policies/artifactreview-badging

${ }^{3}$ [Online]. Available: https://ieeexplore.ieee.org/Xplorehelp/\#/faqs/codeocean\#what-is-ieee-xplore-code-ocean

${ }^{4}$ [Online]. Available: https://ieee-dataport.org/about-ieee-dataport
} 
IEEE DataPort is a universally accessible web-based portal that serves four primary purposes: 1 ) enable individuals and institutions to indefinitely store and make datasets easily accessible to a broad set of researchers, engineers and industry; 2) enable researchers, engineers and industry to gain access to datasets that can be analyzed to advance technology; 3)facilitate data analysis by enabling access to data in the $\mathrm{AWS}^{5}$ Cloud and by enabling the downloading of datasets; and 4)supports reproducible research.

The description concludes with a link between the technology and IEEE's mission:

IEEE DataPort is an online data platform created and supported by IEEE and supports IEEE's overall mission of Advancing Technology for Humanity.

This humanitarian connection is relevant, and we consider it a cornerstone of reproducibility. We go back to it in Section VI-C2.

\section{GRSS Code Library}

The IEEE Remote Sensing Code Library (RSCD) ${ }^{6}$ is

\begin{abstract}
an online curated repository of software related to remote sensing missions, instruments, processing, and applications.

Software should be relevant to the theory, concepts, and techniques of science and engineering as applied to sensing the earth, oceans, atmosphere, and space, and the processing, interpretation, and dissemination of this information.
\end{abstract}

Its contents are reviewed, and accepted contributions receive a digital object identifier (DOI). As such, RSCD is a significant player in the Remote Sensing reproducibility and replicability game.

\section{PROPOSAL}

In this section, we propose a badging system to identify works that comply with minimum requirements of reproducibility and replicability in Remote Sensing research. Fig. 3 shows the workflow for its implementation. This workflow is a functional and updated mapping of the "Compendium" schema proposed by Gentleman and Lang [9].

\section{A. Requirements}

1) Web Page: The first requisite for being considered an RSRR paper is associating the manuscript to a universally accessible and informative web page containing the following.

1) Project identification (one project may host more than one paper; one paper may be hosted by more than one project):
a) title;
b) participants;
c) summary;
d) funding information; and
e) start date, state (in preparation, active, finished).

2) Paper identification (if different from 1):
a) title;
b) contact: the corresponding author;
c) abstract;

\footnotetext{
${ }^{5}$ Amazon Web Services.

${ }^{6}$ [Online]. Available: http://www.grss-ieee.org/publication-category/rscl/
}

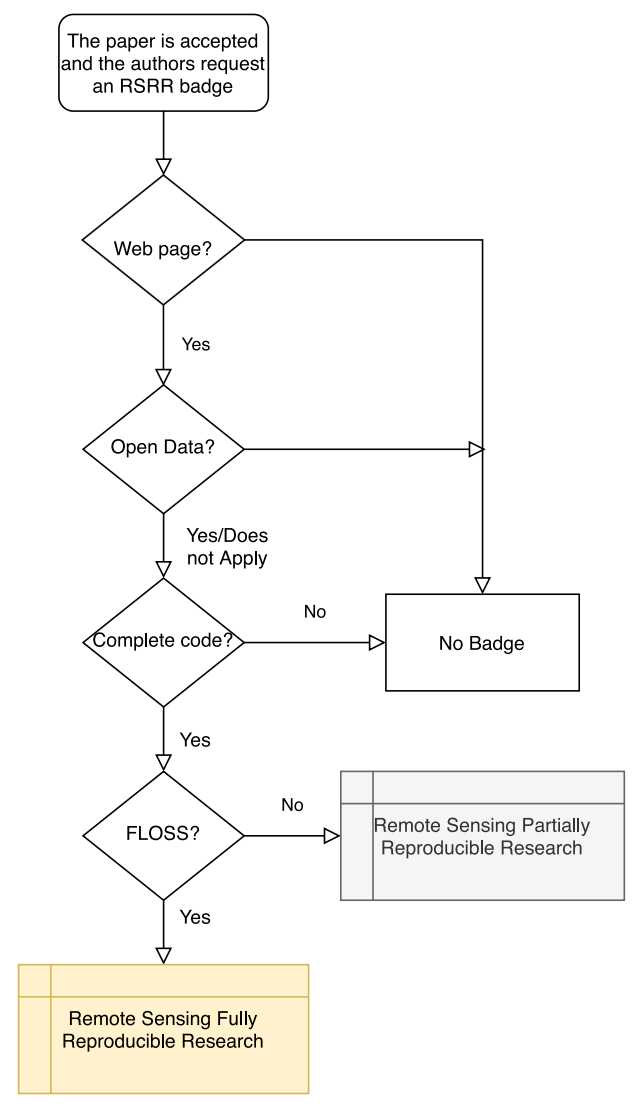

Fig. 3. Flowchart of the badge awarding process.

d) PDFs of relevant versions, including information of its submission to repositories (arXiv, IEEE TechRiv, etc.), journal or conference;

e) ${ }^{\mathrm{A}} \mathrm{T}_{\mathrm{E}} \mathrm{X}$ and $\mathrm{BIBT} \mathrm{T}_{\mathrm{E}} \mathrm{X}$ files, and images, and plots.

3) Computational platform (machine, model, operating system, software, libraries, and versions).

4) Code (with comments).

5) Data.

6) Which software components are FLOSS or free (including operating system)?

7) Provide precise instructions and examples about:

a) how to install and run the code;

b) how to read and modify the data; and

c) how to generate the plots, images (where they improved for visualization? how?), and tables (rounding, truncating, etc.).

Most researchers can produce a basic web page. Bear in mind that basic web pages are likely to attract users, and they suffice to enrich the visibility of the research.

2) Experimental Design: Many studies rely on the information provided by samples; few of them detail the procedure with which they were collected. A reproducible study should, besides providing the samples used, state the following:

1) objective criteria set a priori for sample collection;

2) number of samples, sample size, and descriptive statistics for the aggregated data; and

3) objective criteria for sample selection and data imputation. 
Stochastic simulation is at the core of randomized algorithms, for instance, Monte Carlo experiments. In order to make such algorithms reproducible, apart from the information in Section IV-A1, item 3, the authors must inform the pseudorandom number generator and the seeds employed.

Science is not only made of positive results. Sticking to this path may restrict the outcomes to confirmatory studies, avoiding those lines of research that do not produce immediately publishable results. Scientific honesty requires telling the whole story, starting from clearly defining the research protocol [10]. The research course may change along with the work, but telling the whole story adds more to the scientific knowledge that reporting only the evidence and the conclusions that are aligned with the starting hypotheses.

3) Open Data: Open data can be accessed, used, and shared by Governments, businesses, and individuals. Open data should be stored in widely accessible and persistent repositories, in standard, machine-readable formats. Note that Excel $^{\odot}$ spreadsheets do not comply with this requirement; use comma-separated values (with . CSv extension) instead. Although Open data are not necessarily free data, the authors must at least provide a minimum of free samples and the tools to read and export them to freely available computational platforms.

4) Free/Libre Open Source Software: FLOSS source code is licensed free of charge, encouraging modifications and improvements.

Notable FLOSS platforms for Remote Sensing research are $\mathrm{R}^{7}$ (for statistical computing and production of high-quality graphics), Python ${ }^{8}$ (an interpreted, object-oriented, high-level programming language with dynamic semantics), and GNU Octave $^{9}$ (an array-programming-oriented language). The European Space Agency (ESA) freely distributes SNAP-SeNtinel's Application Platform. ${ }^{10}$ SNAP, developed in Java and Python, is intended for interactive image visualization and analysis, but it also allows scripting and software extension with new functionalities. Moreover, the ESA promotes STEP, a community platform for accessing the software and its documentation, communicating with the developers, dialoguing within the science community, promoting results and achievements, as well as providing tutorials and material for training scientists using the Toolboxes. All platforms mentioned in this section have similar communities.

5) Code: Providing the code that transformed data into knowledge is a cornerstone in RSRR. This sharing requires lots of generosity and a change of mindset, but it is fundamental for the advancement of Science.

Researchers are benefited from the assistance of well-trained programmers (software engineers) that make software's issues simpler. Nevertheless, in other situations, researchers must prepare the codes by themselves, and this is not an easy task. Decisions such as selecting the most appropriate tool (C?, Python?,

\footnotetext{
${ }^{7}$ [Online]. Available: https://www.r-project.org/about.html

${ }^{8}$ [Online]. Available: https://www.python.org/about/

${ }^{9}$ [Online]. Available: https://booki.flossmanuals.net/command-line/gnuoctave.html\#

${ }^{10}$ [Online]. Available: http://step.esa.int/main/toolboxes/snap/
}

on Windows or Linux?) or what public libraries to use are not trivial either.

Therefore, among others, software challengers related to reproducibility and replicability can be summarized as follows:

1) properly select the best software in terms of efficiency, to support the work;

2) properly select the best software to be easily used, updated, and reported; and

3) make the software as easy to use as possible.

Note that software efficiency is closely related to the hardware and operating system on which it runs. Therefore, the implementation must be designed aiming at its easy and fast adaptation to new coming machines and environments. This is especially true for the challenges that the deep learning paradigm brings into Remote Sensing research.

We urge authors to adhere to good practices as, for instance, those discussed in [11, Ch. 7]. Bear in mind that sharing code aims at allowing others to use it, so you must provide running examples and comments that allow your readers to make the necessary changes, for example on how to change the input data.

For instance, for C, Java, Python programmers, the GNU General Public License (GPL) tool, Doxygen, ${ }^{11}$ easily generates documentation from flat sources, and it also provides visual representations of the code in a hierarchical attractive and very useful way.

6) Text: Zobel's book [12] discusses general guidelines of adequate scientific writing. We add elements that promote reproducibility and replicability.

The review of the literature, which allowed the authors to draw the state-of-the-art over which they built their research, should also be reproducible. The systematic review approach aims at being reproducible, auditable, and transparent [13]. Life sciences have expertise in conducting such reviews, while the Remote Sensing community is beginning to adhere to such practice.

The methodological part of the text is essential for reproducibility. It must clearly state all the assumptions, details of the context, materials, and procedures the authors employed during their research. A good Methodology section is a roadmap for reproducing exactly the same study and, ideally, obtaining the same results.

In the same manner, the authors identified missing knowledge while performing the analysis of the state of the art and, with it, made a contribution; their work should point at future enhancements, improvements, and advances. The more straightforward these remarks are, the easier it will be for other researchers (and for the authors themselves) to continuing contributing to this line of research. This analysis belongs to the Discussions section of the manuscript.

Since the source $\mathrm{L}_{\mathrm{E}} \mathrm{T} X$ and $\mathrm{BIBT}_{\mathrm{E}} \mathrm{X}$ will be available, the authors should be generous with comments. Such additional information will not make it to the final published work but can be useful to those readers that will use this material.

7) Additional Artifacts: Current collaborative research usually produces artifacts that may contribute to the replicability

\footnotetext{
${ }^{11}$ [Online]. Available: http://doxygen.nl
} 
of the research. Among them, we may mention Mind Maps, Project Management files, Wikis, and a bug tracking database as Bugzilla. All these elements, well documented, may be added to the paper repository for optional scrutiny and use.

\section{B. Implementation}

GRSS should implement a Remote Sensing Reproducibility Committee for each of its (currently) four journals, namely, IEEE Transactions on Geoscience And REMote SENSING, IEEE GEOSCIENCE AND REMOTE SENSING LETTERS, IEEE Geoscience and Remote Sensing Magazine, and IEEE JOURNAL OF SELECTED TOPICS ON APPLIED EARTH OBSERVATIONS AND REMOTE SENSING. This committee will act solely on accepted papers for which the authors have requested the RSRR Badge. The role of such committee is solely verifying the reproducibility of the article, and thus, the expertise of their members might also be from outside the Remote Sensing community.

The assessment process has three possible outcomes.

1) Fully RSRR: The article is fully compliant with the reproducibility and replicability criteria.

2) Partially RSRR: The article is partially compliant with the reproducibility and replicability criteria, as it does not use exclusively FLOSS software.

3) No badge: The manuscript does not comply with at least one of the mandatory reproducibility requirements.

Fig. 3 illustrates the proposed badge awarding process.

Some of the direct benefits for papers published under the RSRR Badge are as follows.

1) The paper will display the recognition (both for the paper printed and for the online versions).

2) The paper will be specially promoted after publication by the journals.

3) The journal will enforce, in each cross-referenced database, that the paper has that badge recognition.

The work by Santana-Cedrés et al. [14], for instance, qualifies for a Remote Sensing Partially Reproducible Research.

As authors' obligation, a prerequisite to obtaining the badge is the acknowledgment that the paper is reproducible in all the terms for at least five years and that the material has permanent and global identifiers, e.g., DOIs.

\section{TOOLS}

Starting well saves lots of time. We make recommendations that may save time and effort. All of them are either free or offer a free operational version, which usually suits the needs of relatively small RSRR projects.

\section{A. Doodling}

Doodling is an important stage to unlock creativity in a controlled manner. We suggest using FreeMind, ${ }^{12}$ or any other mind map tool, for this purpose.

Fig. 4 shows the mind map product of such a doodling stage. It served as a guide for the structure of this article.

\footnotetext{
${ }^{12}$ [Online]. Available: http://freemind.sourceforge.net/wiki/index.php/
}

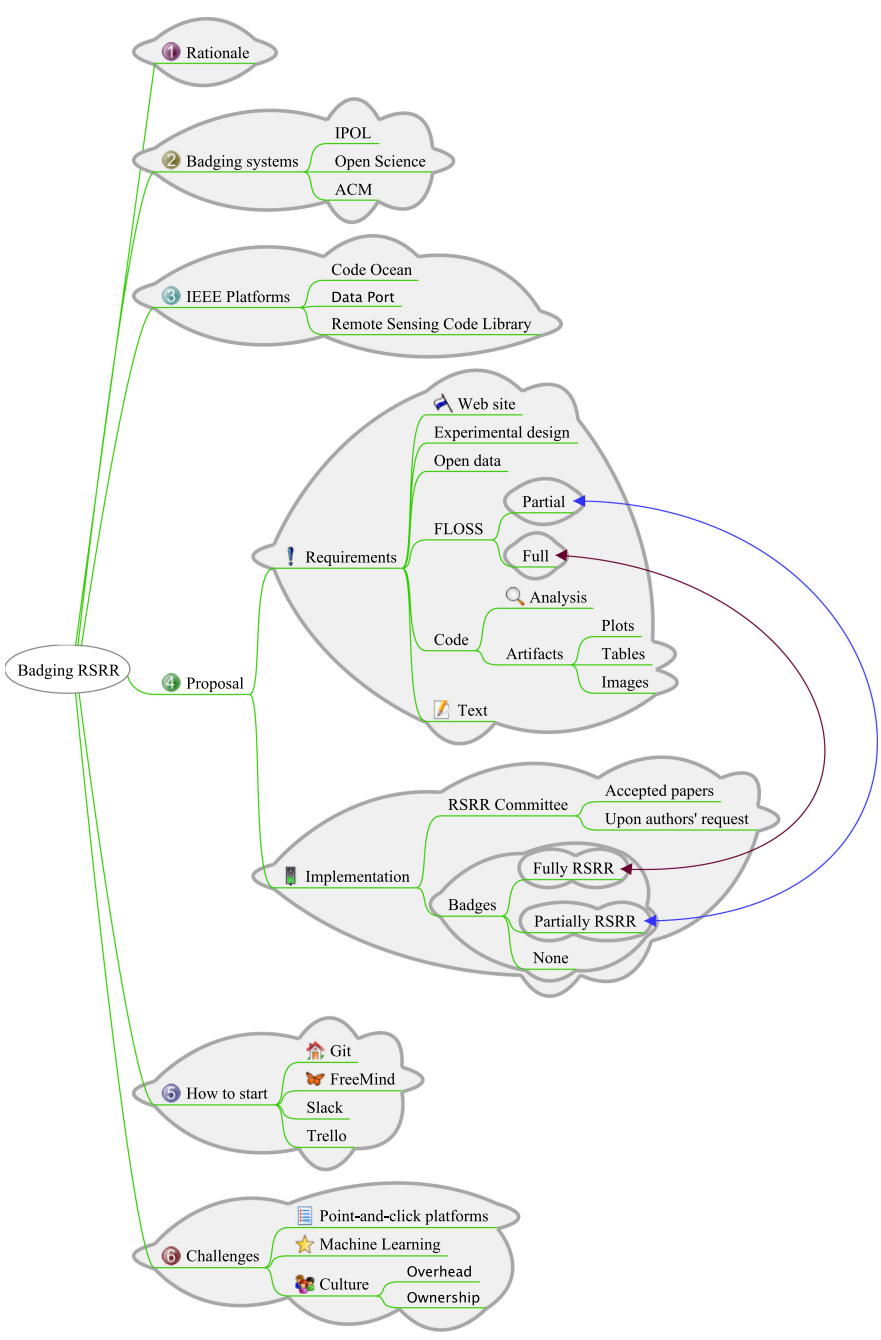

Fig. 4. Mind map that led to this article.

Having such an editable visual structure, along with properties and relationships between elements, helps to remind and documenting the progress of the project.

\section{B. Git Repository}

We assume in this section that the reader uses $\mathrm{L}_{\mathrm{E}} \mathrm{X}$ and BIBTEX.

The main idea consists in having a single repository for all the scientific texts: theses, reports, articles, letters, reviews, and miscellaneous documents.

Fig. 5 illustrates the recommended basic structure for a repository holding several $\mathrm{LAT}_{\mathrm{E} X}$ files (or projects), along with their associated data and code.

Every directory may contain specific subdirectories. For instance, Data may contain CSV, text, and other directories with specific data files.

Note that there is a single $\mathrm{BIBT}_{\mathrm{E}} \mathrm{X}$ file (with extension . bib in the Common directory). $\mathrm{BIBT}_{\mathrm{E}} \mathrm{X}$ references can be split into several files, but these files should be common to all projects. This avoids outdated and redundant bibliographic databases. 


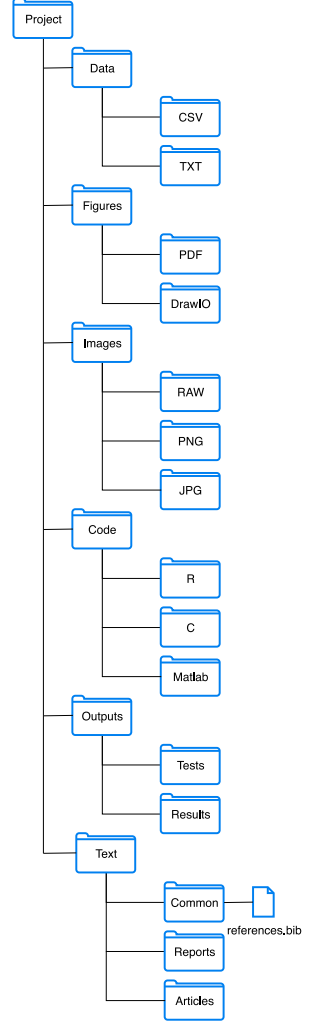

Fig. 5. Recommended structure of a repository for scientific projects.

Every article should be in its directory. Avoid using the name of the journal where the authors will submit the work for the directory and document names, as the destination may change along the process.

Data, figures, images, and code should be common to all projects, as one typically reuses them. Inform in your $\mathrm{LAT}_{\mathrm{E}} \mathrm{X}$ code the source of each figure, for instance with a comment of the form $\% \%$ The source is in ../../Figures / DrawIO/Directorystructure. drawio.

This will help the review process and the communication between authors. All references to source files must use relative paths, e.g., .././more-path/figures, and .././more-path/video_demos. This applies also to codes (. . . . /more-path/my_personal_library).

The Outputs directory should contain both intermediate, but interesting, results (in Tests), and those results that made it into the final report (in Results). Proper documentation is fundamental to help the author, his or her team, and others to catch up from where he or she stopped.

Check [15] for a naming convention and suggestions for revision handling of submitted manuscripts.

\section{Communication}

Most of the research in Remote Sensing is collaborative and involves two or more authors. This is mostly due to the interdisciplinary nature of the area, which requires complementary competencies. Communication in such a scenario is of paramount importance.

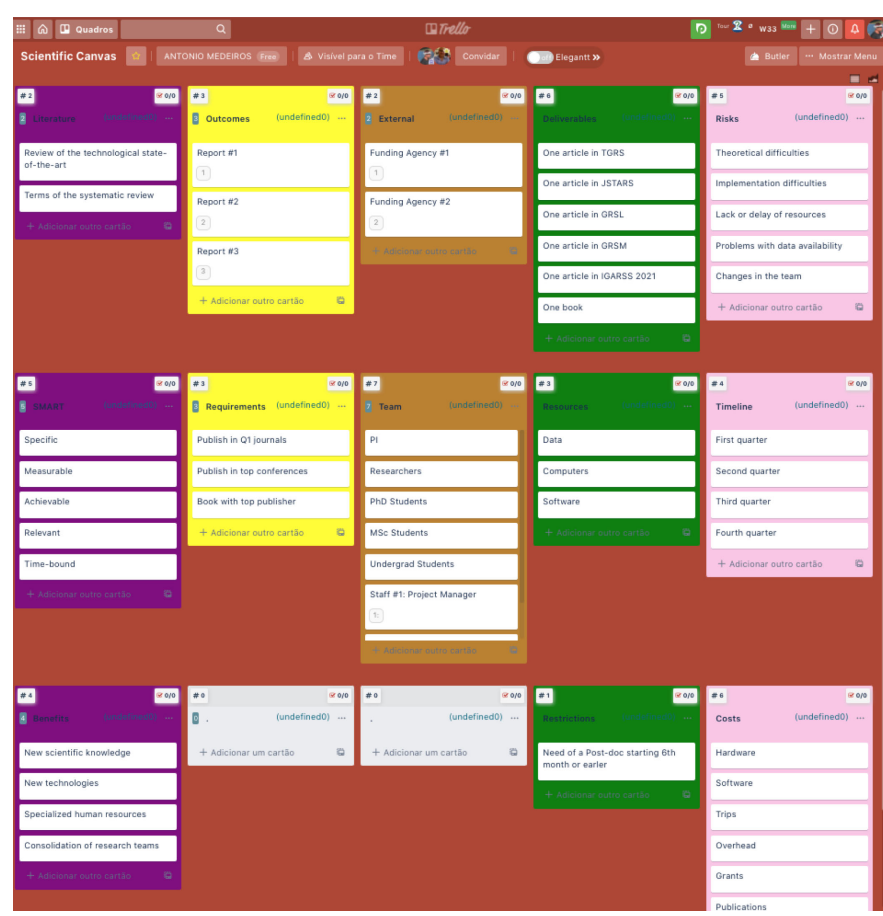

Fig. 6. Trello implementation of a Scientific Canvas.

Although email is a powerful tool, synchronous communication is often required. WhatsApp and WeChat are commonplace for real-time exchange of ideas, but they do not offer either the tools or the organization required for productive and time-saving technical conversations.

Slack ${ }^{13}$ serves this purpose well. It organizes conversations in channels, connects with other applications (Google Suit, meetings, Github, polls, etc.) promoting, thus, focus and persistence.

\section{Task Management}

We have stressed the importance of using a Kanban-style management method for building a project [16]. Such a method is also recommended for simple task management. Trello ${ }^{14}$ usually meets well the needs of organizing and maintaining a Remote Sensing reproducible project. However, a more sophisticated tool, such as ProjectLibre ${ }^{15}$ is highly advisable for projects, which involve several participants, resources, deliverables, and deadlines.

Fig. 6 shows the structure of a Scientific Canvas in Trello, freely available. ${ }^{16}$ Cards are organized in lists, and lists in groups. Each group is identified by a color: "Why?" in purple, "What?" in yellow, "Who?" in brown, "How?" in green, and "When and how much?" in pink. This board can be copied and assigned to a team, whose leader can assign tasks (cards), with deadlines, reminders, and several other utilities. Details of such an organization, which is inspired by the canvases discussed in [17], were presented in [16].

\footnotetext{
${ }^{13}$ [Online]. Available: https://slack.com/

${ }^{14}$ [Online]. Available: https://trello.com/

${ }^{15}$ [Online]. Available: https://www.projectlibre.com/

${ }^{16}$ [Online]. Available: https://trello.com/b/C747M1GX
} 
As Trello keeps track of every single modification, it becomes a useful resource when it comes to reproducibility.

\section{Challenges}

\section{A. Point-and-Click Software}

The ease of use of point-and-click software based on predefined menus is undeniable. Such platforms may conspire against reproducibility, in any case. A fully reproducible process, if based on such a platform, must state the procedure step by step. For this reason, it is advisable to prefer software that allows coding.

\section{B. Machine Learning}

Machine learning, in general, and convolutional neural networks, in particular, have seen an extraordinary increase in popularity in the Remote Sensing community [18] lately. Any reproducible approach must pay special attention to what this so efficient machine learning approach is capable of getting from data (especially from Big Data) [19].

Some of the challenges that these studies pose for their reproducibility are random initialization and the need for GPU resources.

\section{Culture}

1) Overhead: Most experienced researchers frown upon adhering to new practices, especially if they are already successful. It is worth noting that many scientific practices are changing, and adaptation is a sign of intelligence, if not a requirement for survival.

The learning curve of good reproducibility and replicability practices is not steep, and they can be adopted gradually. We have noticed that students and young researchers may be the drivers of this change, which, once in place, facilitates highquality research.

We, thus, consider that overhead is small, limited to the initial stage of the adoption of the practices, and that it returns in the form of better recognition and smoother work.

2) Ownership: Licensing of scientific outcomes is a delicate matter. It grants that authors,' journals,' and Institutions' copyrights. Stodden [20] analyzes some of the possibilities.

Creative Commons licenses ${ }^{17}$ offer to creators specific royalty-free uses for their work. The protection covers the copy and distribution of the material in any medium or format. GNU $\mathrm{GPL}^{18}$ is commonly used nowadays by most CNU programs to protect software (both, source and compiled files).

We conclude reminding that The Universal Declaration of Human Rights, ${ }^{19}$ in its Article 27, states that

Everyone has the right freely to participate in the cultural life of the community, to enjoy the arts and to share in scientific advancement and its benefits.

\footnotetext{
${ }^{17}$ [Online]. Available: https://creativecommons.org/licenses/by-nd/3.0/us/ ${ }^{18}$ [Online]. Available: http://www.gnu.org/licenses/licenses.en.html\#GPL

${ }^{19}$ [Online]. Available: https://www.un.org/en/universal-declaration-humanrights/
}

Adhering to reproducibility and replicability, the Remote Sensing community will give a step in this direction.

This work is available. ${ }^{20}$ The repository can be copied with its contents, or only as a template for other projects.

\section{ACKNOWLEDGMENT}

Disclaimer and Nonaffiliation: The opinions expressed in this presentation are solely those of the presenter and not necessarily those of IEEE, IEEE Geoscience and Remote Sensing Society, and its affiliates. All product and company names are trademarks or registered trademarks of their respective holders. The use of them does not imply any affiliation with or endorsement by them.

\section{REFERENCES}

[1] T. Balz and F. Rocca, "Reproducibility and replicability in SAR remote sensing," IEEE J. Sel. Topics Appl. Earth Observ. Remote Sens., vol. 13 , pp. 3834-3843, 2020.

[2] L. A. Barba, "Terminologies for reproducible research," 2018. [Online]. Available: http://arxiv.org/pdf/1802.03311v1

[3] F. Fidler and J. Wilcox, "Reproducibility of scientific results," in The Stanford Encyclopedia of Philosophy, E. N. Zalta, Ed. Stanford, CA, USA: Metaphysics Research Lab, Stanford University, 2018.

[4] I. Newton and R. Hooke, "Isaac Newton letter to Robert Hooke," 1675. [Online]. Available: https://digitallibrary.hsp.org/index.php/Detail/ objects/9792

[5] L. A. Barba, "Praxis of reproducible computational science," Comput. Sci. Eng., vol. 21, no. 1, pp. 73-78, Jan. 2019.

[6] "Image Processing On Line," Accessed: Sep. 1, 2020. [Online]. Available: http://www.ipol.im/

[7] M. R. Munafò et al. "A manifesto for reproducible science," Nature Human Behav., vol. 1, no. 1, Jan. 2017, Art. no. 0021.

[8] M. Konkol, D. Nüst, and L. Goulier, "Publishing computational researchA review of infrastructures for reproducible and transparent scholarly communication," Res. Integrity Peer Rev., vol. 5, 2020, Art. no. 10.

[9] R. Gentleman and D. T. Lang, "Statistical analyses and reproducible research," Bioconductor Project, The Berkeley Electronic Press, Berkeley, CA, USA, Tech. Rep. Paper 2, 2004

[10] "Tell it like it is," Nature Human Behav., vol. 4, no. 1, p. 1, Jan. 2020

[11] S. Oliveira and D. Stewart, Writing Scientific Software: A Guide to Good Style. Cambridge, U.K.: Cambridge Univ. Press, 2006.

[12] J. Zobel, Writing for Computer Science, 2nd ed. New York, NY, USA: Springer, 2005

[13] D. Tranfield, D. Denyer, and P. Smart, "Towards a methodology for developing evidence-informed management knowledge by means of systematic review," Brit. J. Manage., vol. 14, no. 3, pp. 207-222, Sep. 2003.

[14] D. Santana-Cedrés, L. Gomez, L. Alvarez, and A. C. Frery, "Despeckling PolSAR images with a structure tensor filter," IEEE Geosci. Remote Sens. Lett., vol. 17, no. 2, pp. 357-361, Feb. 2020.

[15] A. C. Frery, "How to successfully make a scientific contribution through IEEE Geoscience and Remote Sensing Letters," IEEE Geosci. Remote Sens. Lett., vol. 12, no. 6, pp. 1167-1169, Jun. 2015.

[16] A. C. Frery, "How to successfully make a scientific contribution BIB foreign language and successful scientific publishing: From the project to the advertising," IEEE Geosci. Remote Sens. Soc. Joint Hyderabad and Bangalore Chapters Webinar, 2020. [Online]. Available: https://ieeemeetings.webex.com/ieeemeetings/ldr.php?RCID= 825b9854c01cd5f07190a96b50284234

[17] A. Osterwalder and Y. Pigneur, Business Model Generation: A Handbook for Visionaries, Game Changers, and Challengers. New York, NY, USA: Wiley, 2010.

[18] M. Reichstein et al.,"Deep learning and process understanding for datadriven Earth system science," Nature, vol. 566, no. 7743, pp. 195-204, Feb. 2019.

\footnotetext{
${ }^{20}$ [Online]. Available: https://acfrery.github.io/Grading-Reproducibility-inRemote-Sensing-Articles/
} 
[19] R. Isdahl and O. E. Gundersen, "Out-of-the-box reproducibility: A survey of machine learning platforms," in Proc. 15th Int. Conf. eSci., Sep. 2019, pp. 86-95.

[20] V. Stodden, "The legal framework for reproducible scientific research: Licensing and copyright," Comput. Sci. Eng., vol. 11, no. 1, pp. 35-40, Jan. 2009.

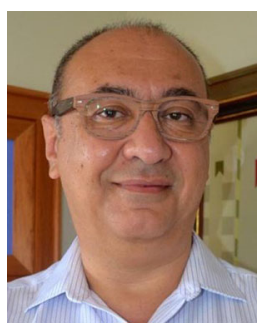

Alejandro C. Frery (Senior Member, IEEE) received the B.Sc. degree in electronic and electrical engineering from the Universidad de Mendoza, Mendoza, Argentina, in 1985, the M.Sc. degree in applied mathematics (statistics) from the Instituto de Matemática Pura e Aplicada, Rio de Janeiro, Brazil, in 1990, and the Ph.D. degree in applied computing from the Instituto Nacional de Pesquisas Espaciais, São José dos Campos, Brazil, in 1993.

He is currently a Professor of Statistics and Data Science with the School of Mathematics and Statistics, Victoria University of Wellington, Wellington, New Zealand, and holds a Huashan Scholar position (2019-2021) with the Key Lab of Intelligent Perception and Image Understanding of the Ministry of Education, Xidian University, Xi' an, China. His research interests include statistical computing and stochastic modeling.

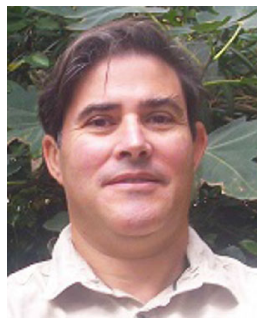

Luis Gomez (Senior Member, IEEE) received the $\mathrm{Ph} . \mathrm{D}$. degree in telecommunication engineering from the University of Las Palmas de Gran Canaria, Las Palmas de Gran Canaria, Spain, in 1992.

Since 1994, he has been an Associate Professor with the Department of Electronic Engineering and Automatic Control, University of Las Palmas de Gran Canaria, where he researches at the Image Technology Center. His current research interests include image processing (denoising, segmentation, and classification) for remote sensing for synthetic aperture radar and polarimetric synthetic aperture radar data.

Dr. Gomez is an Associate Editor for the IEEE GEOsCIENCE AND REMOTE SENSING LETTERS. He is on the Editorial Board of the Image Processing on Line.

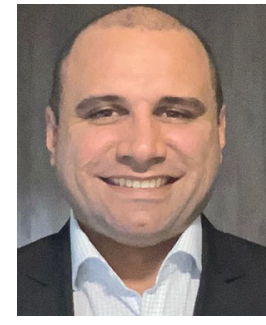

Antonio C. Medeiros received the Graduate degree in meteorology from the Universidade Federal de Alagoas, Maceió, Brazil, in 2016. He is working toward the M.B.A. (Fundação Getúlio Vargas, Rio de Janeiro, Brazil) and M.Sc. (Universidade Federal de Alagoas, Maceió, Brazil) Programs.

$\mathrm{He}$ is currently the Project Manager of the Laboratório de Computaćão Científica e Análise Numérica, Universidade Federal de Alagoas. His research interests include scientific project management, risk assessment, and optimal resource allocation. 